\title{
Femtosecond Laser Micromachining as an Enabling Tool for Optofluidics and Quantum Optics
}

\author{
R. Osellame \\ Istituto di Fotonica e Nanotecnologie (IFN) - CNR \\ Piazza Leonardo da Vinci 32, 20133 Milano, Italy \\ roberto.osellame@ifn.cnr.it
}

\begin{abstract}
The use of femtosecond lasers as microfabrication tools to produce threedimensional photonic circuits and optofluidic networks in transparent materials is reported. Applications to on-chip optical sensing in lab-on-a-chip and integrated quantum circuits will be discussed.
\end{abstract}

Femtosecond lasers are powerful tools for volume microstructuring of transparent materials. Optical waveguide circuits can be directly written without any photolithographic process and exploiting unique three-dimensional capabilities in defining the circuit layout $[1,2]$. In addition, femtosecond-laser irradiation of fused silica followed by chemical etching in HF solution allows the manufacturing of directly buried microfluidic channels, due to the enhanced (by up to two orders of magnitude) etching rate of the irradiated material with respect to the pristine one. This opens the possibility of using a single femtosecond laser system for the production of optical circuits and their possible integration with a microfluidic channel network [3]. This enabling technology has been exploited in such diverse fields as optical detection in lab-on-a-chip and integrated quantum optical circuits.
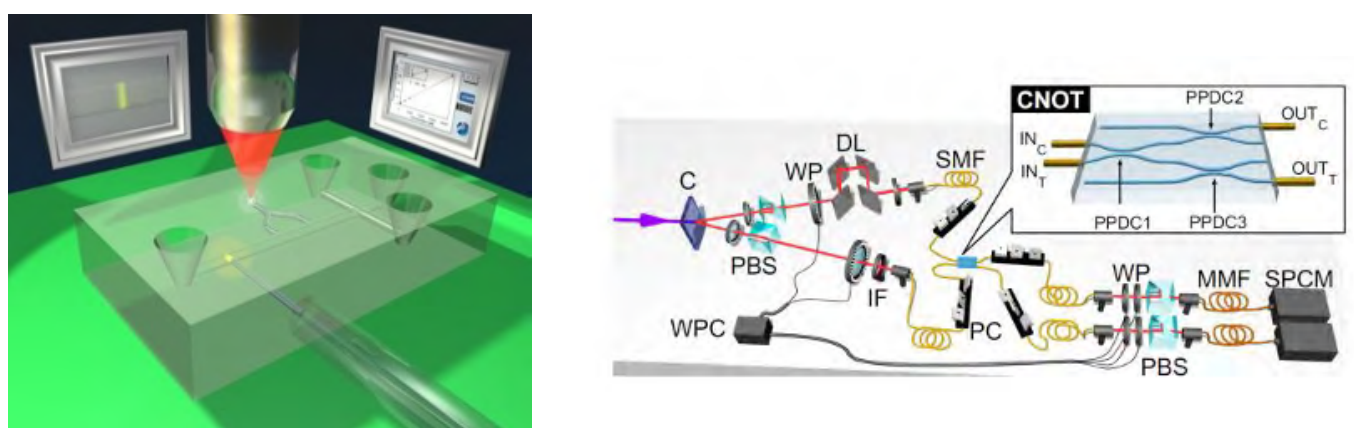

Fig. 1. (Left) Scheme of femtosecond laser direct writing of photonic devices (waveguides, splitters,...) in a lab-on-a-chip for fluorescent sensing of the biomolecules flowing in the microchannel. (Right) Set up for the characterization of a femtosecond laser written optical circuit acting as a CNOT quantum gate for polarization qubits.

A lab-on-chip (LOC) is a device that squeezes onto a single glass substrate the functionalities of a biological laboratory. Femtosecond-laser micromachining is particularly suited for the integration of optical waveguides (or more complex photonic devices such as splitters and interferometers) with a microfluidic network (Fig. 1 left) [4-6]. Applications that will be discussed range from integration of fluorescent [4] and label-free [5] detection in capillary electrophoresis microchips, as well as fabrication of biochips for cell analysis [6].

Quantum optical experiments typically involve several discrete optical elements and thus suffer from complex alignment and instabilities caused by external perturbations. Integrated optical circuits have proven to be a very valuable alternative for robust and scalable quantum optics experiments [7]. Femtosecond laser writing can play a significant role in this field thanks to the rapid prototyping capabilities and to the cost-effective production of small series of devices [8]. Up to now only path entangled photons have been processed in integrated optical circuits, but femtosecond laser writing can also provide low birefringence waveguides which proved to be suitable for quantum information with polarization entangled photons [9]. The realization of a CNOT gate base on polarization qubits will be presented (Fig. 1 right) and perspective of this approach will be discussed.

[1] R.R.Gattass and E. Mazur, "Femtosecond laser micromachining in transparent materials," Nature Photon. 2, 219 (2008).

[2] G. Della Valle, R. Osellame and P. Laporta, "Micromachining of photonic devices by femtosecond laser pulses," J. Opt. A: Pure Appl. Opt. 11, 013001 (2009).

[3] R.Osellame et al., "Integration of optical waveguides and microfluidic channels both fabricated by femtosecond laser irradiation," Appl. Phys. Lett. 90, 231118 (2007).

[4] R.Martinez et al., "Integration of femtosecond laser written optical waveguides in a lab-on-chip," Lab. Chip 9, 91 (2009).

[5] A. Crespi et al., "Three-dimensional Mach-Zehnder interferometer in a microfluidic chip for spatially-resolved label-free detection," Lab. Chip 10, 1167-1173 (2010).

[6] N. Bellini et al., "Femtosecond laser fabricated monolithic chip for optical trapping and stretching of single cells," Opt. Express 18, 4679-4688 (2010).

[7] A. Politi et al., "Silica-on-silicon waveguide quantum circuits," Science 320, 646-649 (2008).

[8] G.D. Marshall et al., "Laser written waveguide photonic quantum circuits," Opt. Express 17, 12546-12554 (2009)

[9] L. Sansoni et al., "Polarization entangled state measurement on a chip," Phys. Rev. Lett. 105, 200503 (2010).

This is an Open Access article distributed under the terms of the Creative Commons Attribution License 2.0, which permits unrestricted use, distribution, and reproduction in any medium, provided the original work is properly cited. 